\title{
Cellular automata and Lyapunov exponents
}

\author{
P. TISSEUR \\ Institut de Mathématiques de Luminy \\ UPR 9016 - 163, avenue de Luminy Case 907 \\ 13288 Marseille Cedex 9 France
}

\begin{abstract}
The first definition of Lyapunov exponents (depending on a probability measure) for a one-dimensional cellular automaton were introduced by Shereshevsky in 1991. The existence of an almost everywhere constant value for each of the two exponents (left and right), requires particular conditions for the measure. Shereshevsky establishes an inequality involving these two constants and the metric entropies of both the shift and the cellular automaton. In this article we first prove that the two Shereshevsky's exponents exist for a more suitable class of measures, then, keeping the same conditions, we define new exponents, called average Lyapunov exponents smaller or equal to the first ones. We obtain two inequalities: the first one is analogous to the Shereshevsky's but concerns the average exponents; the second is the Shereshevsky inequality but with more suitable assumptions. These results are illustrated by two non-trivial examples, both proving that average exponents provide a better bound for the entropy, and one showing that the inequalities are strict in general.
\end{abstract}

\section{Introduction}

A one-dimensional cellular automaton (CA) denote by $F$ is a discrete mathematical idealization of a space-time physical system. The space, called configuration space, consist of a discrete, regular, doubly infinite one-dimensional lattice with the property that each site can take a finite number of different 
values. A configuration is defined when every sites are fixed. The discrete time is represented by the action of a cellular automaton $F$ on this space. This action consist to change the value of a site considering only a finite number of values situated in the neighborhood of this site on the previous time step. We say that we apply a local rule. The definition and the name cellular automaton were first given by Von Neumanmn and Ulam for modeling biological self reproduction.

For differential systems, the Lyapunov exponents are essentially local properties and it is natural to introduce a corresponding definition in the discrete frame of a cellular automaton, defined by a local rule.

A perturbation in the configuration space is intuitively a change of values on some site. In [21, Wolfram call perturbation a change of a finite number of site and study with computer the propagation of these changes. He call Lyapunov exponents the speed of these propagations and suspect that there exists relations between the spatial and temporal entropies and these exponents (see 21] pages 261; 514). The question of these relations appears as one of the 20 general questions raised by Wolfram about cellular automata (see [21] page 172). In [18] Shereshevsky gave a mathematical definition of the Lyapunov exponents for a cellular automaton. A left or right perturbation of a configuration become the set of all the configurations which differ from the first one at the right or left side of the central site. The Shereshevsky definition of the Lyapunov exponents require to take the maximum speed of propagation on all the shifted configurations. Shereshevsky define the left and right Lyapunov exponents maps $\left(\lambda^{+}, \lambda^{-}\right)$(see subsection 3.1) which characterize the speed of propagation of these perturbations with respect to a cellular automaton and shift-invariant measure. Requiring the $F$-ergodicity for the measure he obtains that the maps have almost everywhere the same value and note the two constants $\lambda_{\mu}^{+}$and $\lambda_{\mu}^{-}$. Then if $\mu$ is also shift invariant, denoting by $h_{\mu}(F)$, (resp. $\left.h_{\mu}(\sigma)\right)$ the metric entropy of $F$ (resp. the metric entropy of the shift $\sigma$ ), Shereshevsky establishes an inequality presumed by Wolfram and similar to the Pesin one ( [15] or [16]) in the differentiable case:

$$
h_{\mu}(F) \leq h_{\mu}(\sigma)\left(\lambda_{\mu}^{+}+\lambda_{\mu}^{-}\right)
$$

where $h_{\mu}(F)$ and $h_{\mu}(\sigma)$ are respectively the metric entropy of the cellular automaton $F$ and the metric entropy of the shift $\sigma$.

The main reason for continuing the work of Shereshevsky is that we know very few examples of cellular automaton with F-ergodic measure in general. The only exception are the expansive ones. 
Another reason is that when a cellular automaton has equicontinuous points in the topological support of the measure, the measure can not be F-ergodic. The Proposition 3.1 asserts that these exponents $\lambda_{\mu}^{+}$and $\lambda_{\mu}^{-}$also exist in the case of a shift-ergodic measure which is only $F$-invariant. With these last conditions the uniform measure which is shift-ergodic is also invariant for every onto cellular automata. More generally (see [6]) if $X$ is a mixing subshift of finite type and $F$ a cellular automaton such that $F(X)=X$ then the Parry measure on $X$ verify the new conditions.

From Proposition 3.1, the new measure conditions implies that the exponents $\lambda_{\mu}^{+}$and $\lambda_{\mu}^{-}$only depend on the topological support $S(\mu)$ of $\mu$. To be precise they quantify the maximum speed of the propagation of perturbation on the set $S(\mu)$.

We show by examples that for cellular automata with equicontinuous points in $S(\mu)$ the exponents $\lambda_{\mu}^{+}$and $\lambda_{\mu}^{-}$are strictly positives under the new assumptions (see example 1), although the metric entropie is equal to 0 (see Proposition [5.2).

Next we define new Lyapunov exponents $\left(I_{\mu}^{+}, I_{\mu}^{-}\right)$called average Lyapunov exponents defined respected to an $F$-invariant and shift-ergodic measure.

From Proposition $\mathbf{3 . 2}$, we assume that the new exponents are smaller or equal to the first ones.

They are equal to 0 when exist equicontinuous points (see Proposition $\mathbf{5 . 2}$ and example 1).

Proving that the sum of these two exponents has a sense (see Proposition 5.1) we state the main result named Theorem 5.1 which gives the inequality:

$$
h_{\mu}(F) \leq h_{\mu}(\sigma)\left(I_{\mu}^{+}+I_{\mu}^{-}\right) .
$$

In example 2 we show that the average Lyapunov exponents can be strictly smaller than the Shereshevsky one, even if there is not equicontinuous point. Finally in Proposition 5.3 we establish a topological inequality :

If we denote by $\mu_{u}$ the uniform measure on $A^{\mathbb{Z}}$ and by $h_{\text {top }}(F)$ the topological entropy of an onto cellular automaton $F: A^{\mathbb{Z}} \rightarrow A^{\mathbb{Z}}$ we obtain

$$
h_{\text {top }}(F) \leq \log \# A\left(\lambda_{\mu_{u}}^{+}+\lambda_{\mu_{u}}^{-}\right) .
$$

We underline that when it is useful we only put one synthetic expression $\lambda_{\mu}^{ \pm}$, $\Lambda_{n}^{ \pm}, I_{n}^{ \pm}$, etc. 


\section{Preliminary}

\subsection{Symbolic systems and cellular automata}

Let $A$ be a finite set or alphabet. Denote by $A^{*}$ the set of all concatenations of letters in $A$. These concatenations are called words. The length of a word $u \in A^{*}$ is denoted by $|u|$. The set of bi-infinite sequences $x=\left(x_{i}\right)_{i \in \mathbb{Z}}$ is denoted by $A^{\mathbb{Z}}$. A point $x \in A^{\mathbb{Z}}$ is called a configuration. For $i \leq j$ in $\mathbb{Z}$ we denote by $x(i, j)$ the word $x_{i} \ldots x_{j}$ and by $x(p, \infty)$ the infinite sequence $\left(v_{i}\right)_{i \in \mathbb{N}}$ such that for all $i \in \mathbb{N}$ one has $v_{i}=x_{p+i-1}$. We endow $A^{\mathbb{Z}}$ with the product topology. The shift $\sigma: A^{\mathbb{Z}} \rightarrow A^{\mathbb{Z}}$ is defined by : $\sigma(x)=\left(x_{i+1}\right)_{i \in \mathbb{Z}}$. For each integer $t$ and each word $u$, we call cylinder the set $[u]_{t}=\{x \in$ $\left.A^{\mathbb{Z}}: x_{t}=u_{1} \ldots ; x_{t+|u|}=u_{|u|}\right\}$. For this topology $A^{\mathbb{Z}}$ is a compact metric space. A metric compatible with this topology can be defined by the distance $d(x, y)=2^{-i}$ where $i=\min \{|j|$ such that $x(j) \neq y(j)\}$. The dynamical system $\left(A^{\mathbb{Z}}, \sigma\right)$ is called the full shift. A subshift $X$ is a closed shift-invariant subset $X$ of $A^{\mathbb{Z}}$ endowed with the shift $\sigma$. It is possible to identify $(X, \sigma)$ with the set $X$. A language $L$ is an arbitrary subset of $A^{*}$. Let $L_{n}$ be the set of words of length $n$ of $L$. The language associated to the subshift $X$ is $L(X)=\left\{u \in A^{*} \mid \exists x \in X, x(i, i+|u| w-1)=u\right\}$. It is well known that $(X, \sigma)$ is completely described by $L(X)$. If $\alpha=\left\{A_{1}, \ldots, A_{n}\right\}$ and $\beta=\left\{B_{1}, \ldots, B_{m}\right\}$ are two partitions denote by $\alpha \vee \beta$ the partition $\left\{A_{i} \cap B_{j} i=\right.$ $1 \ldots n, j=1, \ldots, m\}$.

Consider a probability measure $\mu$ on the Borel sigma-algebra $\mathcal{B}$ of $A^{\mathbb{Z}}$. If $\mu$ is $\sigma$-invariant then the topological support of $\mu$ is a subshift denoted by $S(\mu)$. We denote by $\mathcal{M}(\mathcal{F})$ the set of all $F$-invariant probability measures and by $\# A$ the cardinal of the set $\mathrm{A}$. The uniform probability measure on $A^{\mathbb{Z}}$ is the measure such that $\mu\left([u]_{t}\right)=(\# A)^{-k}$ for all integers $t$ and words $u \in A^{k}$. The metric entropy $h_{\mu}(T)$ of a transformation $T$ is an isomorphism invariant between two $\mu$-preserving transformations; its definition can be found in 20] and many other ergodic theory books. A cellular automaton (CA) is a continuous self-map $F$ on $A^{\mathbb{Z}}$ commuting with the shift. The Curtis-Hedlund-Lyndon theorem [7] states that for every cellular automaton $F$ there exist an integer $r$ and a block map $f$ from $A^{2 r+1}$ to $A$ such that: $F(x)_{i}=f\left(x_{i-r}, \ldots, x_{i}, \ldots, x_{i+r}\right)$. The integer $r$ is called the radius of the cellular automaton. If the block map of a cellular automaton is such that $F(x)_{i}=f\left(x_{i}, \ldots, \ldots, x_{i+r}\right)$, the cellular automaton is called one-sided and can be extended a map on a two-sided shift $A^{\mathbb{Z}}$ or a map on a one-sided shift 
$A^{\mathbb{N}}$. If $X$ is a subshift of $A^{\mathbb{Z}}$ and one has $F(X) \subset X$, the restriction of $F$ to $X$ determines a dynamical system $(X, F)$; it is called a cellular automaton on $X$.

\section{$3 \quad$ Lyapunov exponents with shift-ergodic and $F$-invariant measure}

\subsection{The information propagation map}

Consider a cellular automaton $(X, F)$ where $X$ is a subshift of $A^{\mathbb{Z}}$. Set $W_{s}^{+}(x)=\left\{y \in X \mid \forall i \geq s ; y_{i}=x_{i}\right\}$ and $W_{s}^{-}(x)=\left\{y \in X \mid ; i \leq s y_{i}=x_{i}\right\}$. We claim that $W_{s}^{+}(x)$ is the set of perturbations made by infinite blocks of points of $X$ located in the negative coordinates of $x$. For any integer $n$ and $x$ in $X$ one has

$$
\begin{aligned}
& \tilde{\Lambda}_{n}^{+}(x)=\min \left\{s \geq 0: \forall 1 \leq i \leq n, F^{i}\left(W_{0}^{+}(x)\right) \subset W_{s}^{+}\left(F^{i}(x)\right)\right\} \\
& \tilde{\Lambda}_{n}^{-}(x)=\min \left\{s \geq 0: \forall 1 \leq i \leq n, F^{i}\left(W_{0}^{-}(x)\right) \subset W_{-s}^{-}\left(F^{i}(x)\right)\right\} .
\end{aligned}
$$

Then we define the two shift-invariant $\operatorname{maps} \Lambda_{n}^{ \pm}(x)=\max _{i \in \mathbb{Z}} \tilde{\Lambda}_{n}^{ \pm}\left(\sigma^{i}(x)\right)$.

Remark 1 Clearly $\tilde{\Lambda}_{n}^{+}$and $\tilde{\Lambda}_{n}^{-}$are two continuous functions bounded by $r n$. We have changed a little bit the definition of Shereshevsky (see [18] pages 3) in order to clarify some proofs but this change does not affect the limits of the sequences $\left(\frac{\Lambda_{n}^{ \pm}}{n}\right)_{n \in \mathbb{N}}$.

\subsection{One proof of the existence of $\lambda_{\mu}^{+}$and $\lambda_{\mu}^{-}$when $\mu$ is $\sigma$-ergodic.}

In this section we prove that the limits of $\left(\frac{\Lambda_{n}^{ \pm}}{n}\right)_{n \in \mathbb{N}}$ exist almost everywhere when $\mu$ is $\sigma$-ergodic and $F(S(\mu) \subset S(\mu)$ (more suitable conditions) without using the subadditive ergodic theorem. But with this new condition the maximum Lyapunov exponents are rather topological than measure-theoretic quantities because they only depend on the topological support $S(\mu)$.

Proposition 3.1 If $\mu$ is shift-ergodic and $F(S(\mu)) \subset S(\mu)$, for $\mu$-almost all $x$ in $X \supset S(\mu)$ the limits $\lim _{n \rightarrow \infty} \frac{\Lambda_{n}^{+}(x)}{n}$ and $\lim _{n \rightarrow \infty} \frac{\Lambda_{n}^{-}(x)}{n}$ exist and take 
constant values $\lambda_{\mu}^{+}$and $\lambda_{\mu}^{-}$. Moreover for almost all $x$ one has

$$
\lambda_{\mu}^{ \pm}=\lim _{n \rightarrow \infty} \frac{\Lambda_{n}^{ \pm}(x)}{n}=\lim _{n \rightarrow \infty} \max _{y \in S(\mu)} \frac{\Lambda_{n}^{ \pm}(y)}{n}=\max _{y \in S(\mu)} \limsup _{n \rightarrow \infty} \frac{\Lambda_{n}^{ \pm}(y)}{n} .
$$

Proof: We give only the proof for $\lambda_{\mu}^{+}$. For any $F$-invariant subshift $Y$, denote by $\hat{\Lambda}_{n}^{+}(Y)=\max _{x \in Y} \tilde{\Lambda}_{n}^{+}(x)$.

From [18] we have $\tilde{\Lambda}_{n+m}^{+}(x) \leq \tilde{\Lambda}_{n}^{+}(x)+\tilde{\Lambda}_{m}^{+}\left(\sigma^{\tilde{\Lambda}_{n}^{+}(x)} \circ F^{n}(x)\right)$ which implies that $\left(\hat{\Lambda}_{n}^{+}(Y)\right)_{n \in \mathbb{N}}$ is a subadditive sequence. To finish the proof we need to show that for almost all $x$ we have $\Lambda_{n}^{+}(x)=\hat{\Lambda}_{n}^{+}(S(\mu))$ which implies that for almost all $x$, the sequence $\left(\frac{\Lambda^{+}(x)}{n}\right)$ has a limit.

We show that there exists a set $G$ of full measure such that for any integer $n$, the map $\Lambda_{n}^{+}$is constant on $G$ and the value of this constant is $\hat{\Lambda}_{n}^{+}(S(\mu))$. Let $L(S(\mu))$ be the language associated to $S(\mu)$ and let $u \in L(S(\mu))$ be a word of length $2 r n+r$. Clearly the map $\tilde{\Lambda}_{n}^{+}$is constant on the cylinder $[u]_{0}$. Put

$$
V_{n}=\left\{u \in L_{2 r n+r}(S(\mu)) \text { such that } \tilde{\Lambda}_{n}^{+}\left([u]_{0}\right)=\hat{\Lambda}_{n}^{+}(S(\mu))\right\},
$$

and $G_{n}=\left\{x \in S(\mu) \mid \exists i \in \mathbb{N}\right.$ such that $\left.x(i, i+2 r n+r) \in V_{n}\right\}$.

It is easily seen that for any $x \in G_{n}, \Lambda_{n}^{+}(x)=\hat{\Lambda}_{n}^{+}(S(\mu))$. For any $n \in \mathbb{N}$ the set $G_{n}$ is $\sigma$-invariant. It contains a cylinder $[u]_{0}$ such that $u \in V_{n}$, so $\mu\left(G_{n}\right) \geq \mu\left([u]_{0}\right)>0$ and as $\mu$ is $\sigma$-ergodic, $\mu\left(G_{n}\right)=1$. Then $\mu\left(\cap_{n=1}^{\infty} G_{n}\right)=1$ and the map $\Lambda_{n}^{+}$takes the value $\hat{\Lambda}_{n}^{+}(S(\mu))$ on a set of full measure.

\subsection{Average Lyapunov exponents}

In this section we introduce the average exponents $I_{\mu}^{+}$and $I_{\mu}^{-}$which represent an average rate of propagation along the shift orbit for almost all points of $X$. We are going to show that these two exponents are less than or equal to their homologue $\lambda_{\mu}^{+}$and $\lambda_{\mu}^{-}$.

For any integer $n$, any point $x$, the map $I_{n}^{-}$gives the minimum integer $m$ such that all the perturbations in the right side of $x_{m}$ never move until the central coordinate while the $n$ first iterations ; the exponent $I_{n}^{+}$has a symmetric definition. Formally,

$$
\begin{aligned}
& I_{n}^{-}(x)=\min \left\{s \in \mathbb{N}|\forall 1 \leq i \leq n,| F^{i}\left(W_{s}^{-}(x)\right) \subset W_{0}^{-}\left(F^{i}(x)\right)\right\}, \\
& I_{n}^{+}(x)=\min \left\{s \in \mathbb{N}|\forall 1 \leq i \leq n,| F^{i}\left(W_{-s}^{+}(x)\right) \subset W_{0}^{+}\left(F^{i}(x)\right)\right\} .
\end{aligned}
$$


Remark 2 Clearly $I_{n}^{+}$and $I_{n}^{-}$are two continuous functions bounded by $r n$.

Set $I_{n ; \mu}^{+}=\int_{X} I_{n}^{+}(x) d \mu(x)$ and $I_{n ; \mu}^{-}=\int_{X} I_{n}^{-}(x) d \mu(x)$. The Birkhoff's theorem implies that for almost all $x$ one has $I_{n ; \mu}^{+}=\lim _{n \rightarrow \infty} \sum_{i=-m}^{m} \frac{1}{2 m+1} I_{n}^{+}\left(\sigma^{i}(x)\right)$ and $I_{n ; \mu}^{-}=\lim _{n \rightarrow \infty} \sum_{i=-m}^{m} \frac{1}{2 m+1} I_{n}^{-}\left(\sigma^{i}(x)\right)$.

Definition 3.1 Call average Lyapunov exponents the limits

$$
I_{\mu}^{+}=\liminf _{n \rightarrow \infty} \frac{I_{n ; \mu}^{+}}{n} \text { and } I_{\mu}^{-}=\liminf _{n \rightarrow \infty} \frac{I_{n ; \mu}^{-}}{n} .
$$

Proposition 3.2 If $\mu$ is $\sigma$-ergodic and $F(S(\mu)) \subset S(\mu)$, then $I_{\mu}^{+} \leq \lambda_{\mu}^{+}$and $I_{\mu}^{-} \leq \lambda_{\mu}^{-}$.

Proof: By definition of $I_{n}^{+}(x)$, there exists $i \leq n$ such that $F^{i}\left(W_{-I_{n}^{+}(x)+1}^{+}(x)\right)$ $\not \subset W_{0}^{+}\left(F^{i}(x)\right)$. Hence for all $x$ we have $\tilde{\Lambda}_{n}^{+}\left(\sigma^{-I_{n}^{+}(x)+1}(x)\right) \geq I_{n}^{+}(x)-1$, then $\Lambda_{n}^{+}(x) \geq I_{n}^{+}(x)-1$.

We can write that $\int_{S(\mu)} I_{n}^{+}(x) d \mu(x) \leq \int_{S(\mu)}\left(\Lambda_{n}^{+}(x)+1\right) d \mu(x)$ which implies that

$$
\lim \inf _{n \rightarrow \infty} \frac{I_{n ; \mu}^{+}}{n} \leq \lim \inf _{n \rightarrow \infty} \int_{S(\mu)} \frac{\Lambda_{n}^{+}(x)+1}{n} d \mu(x) .
$$

Then using the dominated convergence theorem we get

$$
I_{\mu}^{+} \leq \int_{S(\mu)} \lim _{n \rightarrow \infty} \frac{\Lambda_{n}^{+}(x)}{n} d \mu(x)=\lambda_{\mu}^{+} .
$$

The proof is the same for $I_{\mu}^{-}$and $\lambda_{\mu}^{-}$.

Question: We do not know examples of sequences $\left(\frac{I_{n ; \mu}^{+}}{n}\right)_{n \in \mathbb{N}}$ and $\left(\frac{I_{n ; \mu}^{-}}{n}\right)_{n \in \mathbb{N}}$ which do not converge. Do they exist?

\section{Equicontinuity and Shannon-McMillan- Breiman theorem}

Definition 4.1 A cellular automaton has equicontinuous points (or Lyapunov stable points) if and only if there exists a point $x$ in $X$ such that for all $\epsilon>0$, there exists $\delta>0$, such that for all $y$ in $X$ with $d(x, y)<\delta$ then $d\left(F^{n}(x), F^{n}(y)\right)<\epsilon$ for any $n$. 
Definition 4.2 Let $F$ be a cellular automaton with radius $r$. $A$ word $B \in$ $A^{2 k+1}$ is called blocking word if for all $x$ in $X$ such that $x(-k, k)=B$, there exists an infinite word sequence $v_{n},\left|v_{n}\right|=2 i+1 \geq r$, such that $F^{n}(x)(-i, i)=$ $v_{n}$ for all $n \in \mathbb{Z}^{*}$.

Remark 3 If $B$ is a blocking word and if a point $x$ verifies $x(-k, k)=B$, then the sequence $F^{n}(x)(-\infty,-i)$ does not depend on $x(k,+\infty)$ because $2 i+1 \geq r$. A blocking word completely disconnects the evolution of the coordinates to its left and to its right. This imply that a point with infinitively many occurrences of a blocking word is an equicontinuous point.

The relation between equicontinuity points and blocking words was established in [12] (see also 4]).

Proposition 4.1 [12] 4 ] A cellular automaton $F$ with radius $r$ acting on a transitive subshift $X$ has equicontinuous points if and only if it has a blocking word.

Let $\alpha$ be a finite partition of $X$, let $T$ be a measurable action on $X$ and $\mu$ be a $T$-invariant measure. Denote by $P_{n, \alpha}^{T}(x)$ the element of the partition $\alpha \vee T^{-1} \alpha \vee \ldots \vee T^{-n} \alpha$ which contains $x$ and $h_{\mu}(T, \alpha)$ the metric entropy of $T$ with respect to the partition $\alpha$.

Theorem 4.1 (Shannon-McMillan-Breiman) If $\mu$ is T-invariant, for almost all $x \lim _{n \rightarrow \infty} \frac{-1}{n} \log \mu\left(P_{n, \alpha}^{T}(x)\right)$ exists and one has

$$
\int_{X} \lim _{n \rightarrow \infty} \frac{-1}{n} \log \mu\left(P_{n, \alpha}^{T}(x)\right) d \mu(x)=h_{\mu}(T, \alpha) .
$$

If $\mu$ is a T-ergodic measure, then for almost all $x$

$$
\lim _{n \rightarrow \infty} \frac{-1}{n} \log \mu\left(P_{n, \alpha}^{T}(x)\right)=h_{\mu}(T, \alpha) .
$$

We give a new version of the Shannon-McMillan-Breiman theorem, in the case of a one to one and onto action $T$, this new Proposition will be used in the proof of the main result.

Proposition 4.2 Let $T$ be a one to one and onto action and $\mu$ an ergodic measure. If we denote by $P_{n, m, \alpha}^{T}(x)$ the element of the partition $\alpha \vee T \alpha \ldots \vee$ $T^{m} \alpha \vee T^{-1} \alpha \ldots \vee T^{-n} \alpha$ which contains $x$, we have

$$
\lim _{n+m \rightarrow \infty} \frac{-1}{n+m} \log \mu\left(P_{n, m, \alpha_{p}}^{T}(x)\right)=h_{\mu}\left(T, \alpha_{p}\right) .
$$


We only give a sketch of the proof, the complete proof appears in [19]. Sketch of the proof: (see [14] in which the similar proof for Theorem 4.1] appears)

Let $i(\alpha)(x)=-\log \mu\left(P_{\alpha}^{T}(x)\right)$ and $i(\alpha / \beta)=-\sum_{A \in \alpha} \log \mu(A / \beta)(x) \chi_{A}(x)$. We use the next definition for the metric entropy

$$
h_{\mu}(\alpha, T)=\lim _{n \rightarrow \infty} \int_{X} i\left(\alpha / \vee_{k=1}^{n} T^{k} \alpha\right)(x) d \mu(x) .
$$

Using the "two sided" version of the Birkhoff theorem $\lim _{n+m \rightarrow \infty} \sum_{k=-m}^{n} f \circ$ $T^{k}(x)=\int_{X} f(x) d \mu(x)$ with $f=\lim _{n \rightarrow \infty} i\left(\alpha / \vee_{k=1}^{n} T^{-k} \alpha\right)$ and showing that $P_{n, m, \alpha_{p}}^{T}(x)=i\left(\vee_{k=-n}^{m} T^{k} \alpha\right)(x)=\sum_{k=-m}^{n-1} i\left(\alpha / \vee_{j=1}^{n-k} T^{-j} \alpha\right) \circ T^{k}+i\left(T^{m-1} \alpha\right)$ we obtain

$$
\lim _{n+m \rightarrow \infty} i\left(\vee_{k=-n}^{m} T^{k} \alpha\right)(x)=\lim _{n \rightarrow \infty} \int_{X} i\left(\alpha / \vee_{k=1}^{n} T^{k} \alpha\right)(x) d \mu(x)
$$

\section{Main results}

The proof of our principal result, Theorem 5.1 relies on two propositions and two lemmas. Proposition 5.1 establishes that one can treat independently the perturbations coming from the right and the perturbations coming from the left: this allows to sum the two exponents. Lemma 5.1 permits to split the general proof into two cases, Lemma 5.2 solves the first case and Proposition 5.2 solves the second.

Proposition 5.1 For any triple of positive integers $(n, p, i)$ with $i \leq n$ and $p \geq r$ and for every $x$ in $X$, one has

$$
F^{i}\left(C_{-p-I_{n}^{+}\left(\sigma^{-p}(x)\right)}^{p+I^{-}\left(\sigma^{p}(x)\right)}(x)\right) \subset C_{-p}^{p}\left(F^{i}(x)\right) .
$$

This means that if the point $y$ has the same coordinate as $x$ from $-p-$ $I_{n}^{+}\left(\sigma^{p}(x)\right)$ to $p+I_{n}^{-}\left(\sigma^{p}(x)\right)$, then for each $i \leq n$ the coordinates of $F^{i}(y)$ are equal of those of $F^{i}(x)$ from $-p$ to $p$.

Proof: Fix two positives integers $n$ and $p$. Choose a point $x \in X$ and put $s^{+}=I_{n}^{+}\left(\sigma^{-p}(x)\right)$ and $s^{-}=I_{n}^{-}\left(\sigma^{p}(x)\right)$. For each point $y \in C_{-p-s^{+}}^{p+s^{-}}(x)$, set $y_{1}$ and $y_{2}$ such that $y_{1}\left(-p-s^{+}, \infty\right)=x\left(-p-s^{+}, \infty\right), y_{1}\left(-\infty, p+s^{-}\right)=$ $y\left(-\infty, p+s^{-}\right), y_{2}\left(-\infty, p+s^{-}\right)=x\left(-\infty, p+s^{-}\right)$and $y_{2}\left(-p-s^{+}, \infty\right)=y(-p-$ $\left.s^{+}, \infty\right)$. By definition of $I_{n}^{+}$and $I_{n}^{-}$, for all $i \leq n$ one has $F^{i}\left(y_{1}\right)(-p, \infty)=$ 
$F^{i}(x)(-p, \infty)$ and $F^{i}\left(y_{2}\right)(-\infty, p)=F^{i}(x)(-\infty, p)$. The proof consists in showing by induction that for every positive integer $i \leq n$ one has

$$
F^{i}(y)(-p, p)=F^{i}(x)(-p, p) \text {. }
$$

Recall that $f: A^{2 r+1} \rightarrow A$ is the local map of $F$; for every integer $k$ we also denote by $f$ the map from $A^{2 r+1+k}$ to $A^{k}$ define by

$$
f\left(u_{0} \ldots u_{2 r+k+1}\right)=f\left(u_{0} \ldots u_{2 r}\right) f\left(u_{1} \ldots u_{2 r+1}\right) \ldots f\left(u_{k-1} \ldots u_{2 r+k}\right) .
$$

Let us prove the first step of the recurrence. If $p \geq r$ then by $F(y)(-p, p)=$ $F(x)(-p, p)$. As $y_{1}(-p-r, r)=y(-p-r, r)$ and $y_{2}(-r, p+r)=y(-r, p+r)$ one has

$$
\begin{gathered}
F(y)(-p, p)=f(y(-p-r, p+r))=f(y(-p-r, r)) f(y(-r, p+r)) \\
=f\left(y_{1}(-p-r, r)\right) f\left(y_{2}(-r, p+r)\right)
\end{gathered}
$$

using the definition of $y_{1}$ and $y_{2}$ we obtain

$$
F(y)(-p, p)=F(x)(-p, 0) F(x)(0, p)=F(x)(-p, p) .
$$

Let $i$ be a positive integer such that $i \leq n-1$. We show that if (4) is true for each $k \leq i$ it remains true for $k=i+1$.

First we need the two equalities

$$
F^{i}\left(y_{1}\right)(-p-r, r)=F^{i}(y)(-p-r, r) \text { and } F^{i}\left(y_{2}\right)(-r, p+r)=F^{i}(y)(-r, p+r) .
$$

We give the proof of the first one, the second is analogous.

We prove the equality $F^{i}\left(y_{1}\right)(-p-r, r)=F^{i}(y)(-p-r, r)$ using a secondary recurrence that establishes that for any positive integer $k \leq i$ one has

$$
F^{k}\left(y_{1}\right)(-p-r(i+1-k), r)=F^{k}(y)(-p-r(i+1-k), r) .
$$

As $y_{1}\left(-p-(r+1) i, p+s^{-}\right)=y\left(-p-(r+1) i, p+s^{-}\right)$, from the definition of $s^{-}$ one has $F\left(y_{1}\right)(-p-r i, p)=F(y)(-p-r i, p)$ and since $p \geq r$ we obtain the first step of this new recurrence. We suppose (6) is true for each $k \leq i-1$, i.e., $F^{k}\left(y_{1}\right)(-p-r(i+1-k), r)=F^{k}(y)(-p-r(i+1-k), r)$; then

$$
F^{k+1}\left(y_{1}\right)(-p-r(i-k), r)=f\left(F^{k}\left(y_{1}\right)(-p-r(i+1-k), 2 r)\right)
$$




$$
=f\left(F^{k}\left(y_{1}\right)(-p-r(i+1-k), r) f\left(F^{k}\left(y_{1}\right)(-r, 2 r)\right) .\right.
$$

and since (6) is true for each $k \leq i-1$ we have

$$
\begin{gathered}
F^{k+1}\left(y_{1}\right)(-p-r(i-k), r)=f\left(F^{k}(y)(-p-r(i+1-k), r)\right) f\left(F^{k}\left(y_{1}\right)(-r, 2 r)\right) \\
=F^{k+1}(y(-p-r(i-k), 0)) f\left(F^{k}\left(y_{1}\right)(-r, 2 r)\right) .
\end{gathered}
$$

To finish the proof of the step $k+1$ of this secondary recurrence, i.e., $F^{k+1}\left(y_{1}\right)(-p-r(i-k), r)=F^{k+1} k(y)(-p-r(i-k), r)$, we need to verify that $f\left(F^{k}\left(y_{1}\right)(-r, 2 r)\right)=f\left(F^{k}(y)(-r, 2 r)\right)=F^{k+1}(y)(0, r)$. From the definition of $y_{1}$ we can assert that $F^{k}\left(y_{1}\right)(-r, 2 r)=F^{k}(x)(-r, 2 r)$ and if we use the hypothesis of the main recurrence (6), namely, $F^{k+1}(y)(-p, p)=$ $F^{k+1}(x)(-p, p)($ since $k \leq i-1)$, we conclude that

$$
\begin{aligned}
f\left(F^{k}\left(y_{1}\right)(-r, 2 r)\right) & =f\left(F^{k}(x)(-r, 2 r)\right)=F^{k+1}(x)(0, r) \\
& =F^{k+1}(y)(0, r) .
\end{aligned}
$$

We are now in position to show that $F^{i+1}(y)(-p, p)=F^{i+1}(x)(-p, p)$, which completes the proof of the main recurrence. Starting from the equality

$F^{i+1}(y)(-p, p)=f\left(F^{i}(y)(-p-r, p+r)\right)=f\left(F^{i}(y)(-p-r, r)\right) f\left(F^{i}(y)(-r, p+r)\right)$

and using the two equalities (5) we conclude that

$$
\begin{aligned}
& F^{i+1}(y)(-p, p) \\
& =f\left(F^{i}\left(y_{1}\right)(-p-r, r)\right) f\left(F^{i}\left(y_{2}\right)(-r, p+r)\right) \\
& =f\left(F^{i}(x)(-p-r, r)\right) f\left(F^{i}(x)(-r, p+r)\right) \\
& =F^{i+1}(x)(-p, 0) F^{i+1}(x)(0, p) \\
& =F^{i+1}(x)(-p, p) .
\end{aligned}
$$

Lemma 5.1 Let $\mu$ be a shift-ergodic measure. If there exist an equicontinuous point $x$ in $S(\mu)$ then for every integer $p \geq r$ and for almost all point the sequences $\left(I_{n}^{+}\left(\sigma^{-p}(x)\right)+I_{n}^{-}\left(\sigma^{p}(x)\right)\right)_{n \in \mathbb{N}}$ are bounded.

If there is not equicontinuous point in $S(\mu)$ then for every $p \geq r$ and for almost all points $x$, the sequences $\left(I_{n}^{+}\left(\sigma^{-p}(x)\right)+I_{n}^{-}\left(\sigma^{p}(x)\right)\right)_{n \in \mathbb{N}}$ go to infinity. 
Proof: If exist an equicontinuous point $x$ in $S(\mu)$ then there exist an integer $k$ and a blocking word $B=x(-k, k)$ (see Proposition 4.1). Let $V(B)$ be the set of all the point with infinitely many occurrences of $B$ in the positive and negative coordinates. From Remark 3 we claim that all the point of $V(B)$ are equicontinuous points. As $\mu$ is shift-ergodic and $\mu\left([B]_{0}\right)>0$ then one has $\mu(V(B))=1$. Using Remark 3 we conclude that for each point $y \in V(B)$ and for each integer $p$ the sequences $\left(I_{n}^{+}\left(\sigma^{-p}(y)\right)+I_{n}^{-}\left(\sigma^{p}(y)\right)\right)_{n \in \mathbb{N}}$ are bounded.

We suppose now that there is not equicontinuous point in $S(\mu)$ and that there exist a set $E$ with strictly positive measure such that $E$ contains only points $x$ with the properties $\exists p(x) \in \mathbb{N} \mid\left(I_{n}^{+}\left(\sigma^{-p}(x)\right)+I_{n}^{-}\left(\sigma^{p}(x)\right)\right)_{n \in \mathbb{N}}$ is a bounded sequence.

Clearly $E \cap S(\mu) \neq \emptyset$. Let $y \in E \cap S(\mu)$. There exist $p \geq r$ such that $M(+)=\max _{n \in \mathbb{N}}\left\{I_{n}^{+}\left(\sigma^{-p}(y)\right)\right\}$ and $M(-)=\max _{n \in \mathbb{N}}\left\{I_{n}^{-}\left(\sigma^{p}(y)\right)\right\}$ are well defined. From Proposition 5.1 for all $i \in \mathbb{N}$ one has

$$
F^{i}\left(C_{-p-M(-)}^{p+M(+)}(y)\right) \subset C_{-p}^{p}\left(F^{i}(y)\right) .
$$

which implies that the word $B^{\prime}=y_{-M(-)-p}, \ldots y_{M(+)+p}$ is a blocking word for $F$. As $\mu$ is shift-ergodic and $\mu\left(\left[B^{\prime}\right]_{0}\right)>0$ then there exist a point $z \in$ $S(\mu)$ with infinitely many occurrences of $B^{\prime}$ in the positive and negative coordinates. This point $z$ is an equicontinuous point (see Remark 3) which contradict the hypothesis.

Lemma 5.2 If $\mu$ is a shift-ergodic and $F$-invariant measure such that $F$ has no equicontinuous point in $S(\mu)$, then $h_{\mu}(F) \leq h_{\mu}(\sigma)\left(I_{\mu}^{+}+I_{\mu}^{-}\right)$.

Proof: Fix $x \in X$ and denote by $\alpha_{p}$ the partition of $X$ into cylinders $C_{-p}^{p}$ $(x \in X)$; call $P_{n, \alpha_{p}}^{F}(x)$ the element of the partition $\alpha_{p} \vee F^{-1} \alpha_{p} \vee \ldots \vee F^{-n} \alpha_{p}$ that contains $x$. By Lemma 5.1, for any choice of positive integers $p(p \geq r)$, $n$ and $i(i \leq n)$, one has

$$
\left.F^{i}\left(C_{-p-I_{n}^{+}\left(\sigma^{-p}(x)\right)}^{p+I_{n}^{-}\left(\sigma^{p}(x)\right.}\right)\right) \subset C_{-p}^{p}\left(F^{i}(x)\right) .
$$

The last inclusion implies that each $F^{-i} \alpha_{p}$ has an element that contains the cylinder $C_{-p-I_{n}^{+}\left(\sigma^{-p}(x)\right)}^{p+I_{n}^{-}\left(\sigma^{p}(x)\right)}(x)$, so $P_{n, \alpha_{p}}^{F}(x) \supset C_{-p-I_{n}^{+}\left(\sigma^{-p}(x)\right)}^{p+I_{n}^{-}\left(\sigma^{p}(x)\right)}(x)$, and consequently

$$
-\frac{1}{n} \log \mu\left(P_{n, \alpha_{p}}^{F}(x)\right) \leq-\frac{1}{n} \log \mu\left(C_{-p-I_{n}^{+}\left(\sigma^{-p}(x)\right)}^{p+I_{n}^{-}\left(\sigma^{p}(x)\right)}\right) .
$$


Applying the Shannon-McMillan-Breiman theorem to $F$ one shows that $h_{\mu}\left(F, \alpha_{p}\right)=\int_{X} \lim _{n \rightarrow \infty}-\frac{1}{n} \log \mu\left(P_{n, \alpha_{p}}^{F}(x)\right)$. Then by $(7)$

$$
\begin{gathered}
h_{\mu}\left(F, \alpha_{p}\right) \leq \int_{X} \liminf _{n \rightarrow \infty}-\frac{1}{n} \log \mu\left(C_{-p-I_{n}^{+}\left(\sigma^{-p}(x)\right)}^{p+I_{n}^{-}\left(\sigma^{p}(x)\right)}(x) d \mu(x)\right. \text { and } \\
h_{\mu}\left(F, \alpha_{p}\right) \leq \int_{X} \liminf _{n \rightarrow \infty}-\frac{\log \mu\left(C_{-p-I_{n}^{+}\left(\sigma^{-p}(x)\right)}^{\left.p+I^{-}(x)\right)}\right.}{I_{n}^{+}\left(\sigma^{-p}(x)\right)+I_{n}^{-}\left(\sigma^{p}(x)\right)} \\
\times \frac{I_{n}^{+}\left(\sigma^{-p}(x)\right)+I_{n}^{-}\left(\sigma^{p}(x)\right)}{n} d \mu(x) .
\end{gathered}
$$

By Lemma 5.1 if there is no equicontinuous point in $S(\mu)$ then for all integer $p$ and almost every point $x$ the sequence $\left(I_{n}^{+}\left(\sigma^{-p}(x)\right)+I_{n}^{-}\left(\sigma^{p}(x)\right)\right)_{n \in \mathbb{N}}$ goes to infinity. Considering that $\mu$ is shift-ergodic and $\sigma$ is a one-to-one and onto map we can apply to $\sigma$ the version (3) of the Shannon-McMillan-Breiman theorem, which gives

$$
\lim _{n \rightarrow \infty}-\frac{\log \mu\left(C_{-p-I_{n}^{+}\left(\sigma^{-p}(x)\right)}^{p+I_{n}^{-}\left(\sigma^{p}(x)\right)}\right)}{I_{n}^{+}\left(\sigma^{-p}(x)\right)+I_{n}^{-}\left(\sigma^{p}(x)\right)}=h_{\mu}\left(\sigma, \alpha_{p}\right)=h_{\mu}(\sigma)
$$

for almost all $x$ and every positive integer $p$. Combining the last equality with (8) yields

$$
h_{\mu}\left(F, \alpha_{p}\right) \leq h_{\mu}(\sigma) \times \int_{X} \liminf _{n \rightarrow \infty} \frac{I_{n}^{+}\left(\sigma^{-p}(x)\right)+I_{n}^{-}\left(\sigma^{p}(x)\right)}{n} d \mu(x) .
$$

Using the Fatou lemma, we get

$$
h_{\mu}\left(F, \alpha_{p}\right) \leq h_{\mu}(\sigma) \times \liminf _{n \rightarrow \infty} \int_{X} \frac{I_{n}^{+}\left(\sigma^{-p}(x)\right)+I_{n}^{-}\left(\sigma^{p}(x)\right)}{n} d \mu(x) .
$$

Since $\alpha_{p}$ is an increasing sequence with the property $\bigvee_{0}^{\infty} \alpha_{i}=\mathcal{B}$ and $\mu$ is $\sigma$-invariant we obtain

$$
\lim _{p \rightarrow \infty} h_{\mu}\left(F, \alpha_{p}\right)=h_{\mu}(F) \leq h_{\mu}(\sigma) \times \liminf _{n \rightarrow \infty} \int_{X} \frac{I_{n}^{+}(x)+I_{n}^{-}(x)}{n} d \mu(x) .
$$

This last inequality completes the proof, so $h_{\mu}(F) \leq h_{\mu}(\sigma) \times\left(I_{\mu}^{+}+I_{\mu}^{-}\right)$.

The next proposition establishes that if there exists a blocking word $u$ such that $\mu\left([u]_{0}\right)>0$ then the metric entropy $h_{\mu}(F)$ is equal to 0 . 
Proposition 5.2 If a cellular automaton $F$ has equicontinuous points belonging to $S(\mu)$ then the average Lyapunov exponents $I_{\mu}^{+}$and $I_{\mu}^{-}$and the metric entropy $h_{\mu}(F)$ are 0 .

Proof: By our hypothesis and Lemma 5.1 for each integer $p \geq r$ the sequences $\left(I_{n}^{+}\left(\sigma^{-p}(x)\right)+I_{n}^{-}\left(\sigma^{p}(x)\right)\right)_{n \in \mathbb{N}}$ are bounded for almost all $x$. This implies that for any positive integer $p$ and for almost all $x$,

$$
\liminf _{n \rightarrow \infty}-\frac{\log \mu\left(C_{-p-I_{n}^{+}\left(\sigma^{-p}(x)\right)}^{p+I_{n}^{-}\left(\sigma^{p}(x)\right)}\right)}{I_{n}^{+}\left(\sigma^{-p}(x)\right)+I_{n}^{-}\left(\sigma^{p}(x)\right)}
$$

is bounded. Then for all $p$ and for almost all $x$, we get

$$
\liminf _{n \rightarrow \infty} \frac{-\log \mu\left(C_{-p-I_{n}^{+}\left(\sigma^{-p}(x)\right)}^{p+I_{-}^{-}\left(\sigma^{p}(x)\right)}\right)}{I_{n}^{+}\left(\sigma^{-p}(x)\right)+I_{n}^{-}\left(\sigma^{p}(x)\right)} \times \frac{I_{n}^{+}\left(\sigma^{-p}(x)\right)+I_{n}^{-}\left(\sigma^{p}(x)\right)}{n}=0 .
$$

From (8) in the proof of Lemma 5.2, the sum over $X$ of the last equality is an upper bound of the metric entropy $h_{\mu}(F)$ which implies that this entropy is equal to 0 . On the other hand the sequence $\left(\frac{I_{n}^{+}(x)}{n}\right)_{n \in \mathbb{N}}$ is bounded by $r$ and converges to 0 for almost all $x$, then applying the dominated convergence theorem one gets

$$
I_{\mu}^{+}=\lim _{n \rightarrow \infty} \int_{X} \frac{I_{n}^{+}(x)}{n} d \mu(x) \leq \int_{X} \lim _{n \rightarrow \infty} \frac{I_{n}^{+}(x)}{n} d \mu(x)=0 .
$$

The proof is identical for $I_{\mu}^{-}$.

Remark 4 One can prove that $h_{\mu}(F)=0$ if there exist equicontinuous points in $S(\mu)$ using Katok's definition of metric entropy.

Combining Lemma 5.2 and Proposition 5.2 we obtain the next theorem :

Theorem 5.1 If $\mu$ is a $\sigma$-ergodic and F-invariant measure then

$$
h_{\mu}(F) \leq h_{\mu}(\sigma)\left(I_{\mu}^{+}+I_{\mu}^{-}\right) .
$$

Remark 5 For one-sided cellular automata one defines a unique average Lyapunov exponent $I_{\mu}$ whose definition is identical to that of $I_{\mu}^{+}$in this 
Subsection. Then the proof of the inequality $h_{\mu}(F) \leq h_{\mu}(\sigma) I_{\mu}$ does not require the use of Proposition 5.1.

Since $\lambda_{\mu}^{+} \geq I_{\mu}^{+}$and $\lambda_{\mu}^{-} \geq I_{\mu}^{-}$(Lemma 3.2) ) one has

Corollary 5.1 If $\mu$ is a $\sigma$-ergodic and $F$-invariant measure then

$$
h_{\mu}(F) \leq h_{\mu}(\sigma)\left(\lambda_{\mu}^{+}+\lambda_{\mu}^{-}\right) .
$$

\section{A topological inequality}

Here we recall some definitions relative to the topological entropy that we denote by $h_{t o p}(F)$. Let $(X, F)$ be a dynamical system. For any integer $n$ the distance $d_{n}$ is defined by $\forall x, y \in X^{2}$ one has $d_{n}(x, y)=\max \left\{d\left(F^{i}(x), F^{i}(y)\right) 0 \leq\right.$ $i \leq n\}$. An $(n, \epsilon)$-covering set is a cover of $X$ by balls of diameter $\epsilon$ for the $d_{n}$ metric. Let $D(n, \epsilon)$ be the minimum cardinal of an $(n, \epsilon)$ covering set.

$$
h_{\text {Top }}(F)=\lim _{\epsilon \rightarrow \infty} \lim _{n \rightarrow \infty} \frac{1}{n} \log (D(n, \epsilon)) .
$$

Let $\mu_{u}$ be the uniform measure of $A^{\mathbb{Z}}$. We will give a upper bound of $h_{\text {top }}(F)$ according to the exponents $\lambda_{\mu_{u}}^{+}$and $\lambda_{\mu_{u}}^{-}$. Remark that for all cellular automaton $F$, the uniform measure satisfies the two conditions of Proposition 3.1. so $\lambda_{\mu_{u}}^{+}$and $\lambda_{\mu_{u}}^{-}$always exist.

Proposition 5.3 For any onto cellular automaton $F: A^{\mathbb{Z}} \rightarrow A^{\mathbb{Z}}$ one has $h_{\text {top }}(F) \leq\left(\lambda_{\mu_{u}}^{+}+\lambda_{\mu_{u}}^{-}\right) \log \# A$.

Proof : From Proposition [5.1 and proof of Proposition $3.2\left(\Lambda_{n}^{ \pm}(x)+1 \geq\right.$ $\left.I_{n}^{ \pm}(x)\right)$, it follows that for any choice of positive integers $p(p \geq r), n$ and $i$ $(i \leq n)$, one has

$$
F^{i}\left(C_{-p-\Lambda_{n}^{-}(x)-1}^{p+\Lambda_{n}^{+}(x)+1}(x)\right) \subset C_{-p}^{p}\left(F^{i}(x)\right) .
$$

Denote by $\Omega(n, p))$ the set of all the cylinders $\left(C_{-p-\Lambda_{n}^{-}(x)-1}^{p+\Lambda_{n}^{+}(x)+1}(x)\left(x \in A^{\mathbb{Z}}\right)\right)$ and by $\hat{\Lambda}_{n}^{ \pm}$the maximum of all the $\Lambda_{n}^{ \pm}(x)$. The last inequality implies that $\Omega(n, p))$ is a $\left(n, 2^{-p}\right)$ covering set which show that for all integers $n$ and $p$, we get $D\left(n, 2^{-p}\right) \leq \# \Omega(n, p)$. As

$$
\# \Omega(n, p)=\#\left\{C_{-p-\hat{\Lambda}_{n}^{+}-1}^{p+\hat{\Lambda}_{-}^{-}+1}\left(x_{j}\right) \mid x_{j} \in A^{\mathbb{Z}}\right\}=(\# A)^{\left(2 p+3+\hat{\Lambda}_{n}^{+}+\hat{\Lambda}_{n}^{-}\right)},
$$


we can assert that

$$
\begin{aligned}
h_{\text {Top }}(F)=\lim _{p \rightarrow \infty} & \lim _{n \rightarrow \infty} \frac{1}{n} \log D\left(n, 2^{-p}\right) \\
& \leq \limsup _{p \rightarrow \infty} \lim _{n \rightarrow \infty} \frac{2 p+3+\hat{\Lambda}_{n}^{+}+\hat{\Lambda}_{n}^{-}}{n} \times \log \# A .
\end{aligned}
$$

Using Proposition 3.1 we obtain $h_{\text {Top }}(F) \leq\left(\lambda_{\mu_{u}}^{+}+\lambda_{\mu_{u}}^{-}\right) \log \# A$.

\section{Examples}

The two following examples show that $I_{\mu}^{+}$and $I_{\mu}^{-}$can be strictly less than $\lambda_{\mu}^{+}$and $\lambda_{\mu}^{-}$. The example 6.2 shows that the inequality of Theorem 5.1 is in general strict. In both examples we use the uniform measure which is shift-ergodic and $F$-invariant when $F$ is onto from $A^{\mathbb{Z}}$ to itself.

\subsection{Coven's cellular automata}

In [5], Coven computes exactly the positive topological entropy of a particular class of onto cellular automata with complex behavior. In 3] Blanchard and Maass show that all these $\mathrm{CA}$ have equicontinuous points.

A Coven aperiodic CA is defined by its block map $f:\{0,1\}^{r+1} \rightarrow\{0,1\}$ : $f\left(x_{0}, x_{1}, \ldots, x_{r}\right)=\left(x_{0}+1\right) \bmod 2$ if $x_{1} \ldots x_{r}=b_{1} \ldots b_{r}, f\left(x_{0}, x_{1}, \ldots, x_{r}\right)=x_{0}$ otherwise. The word $B=b_{1} \ldots b_{r}$ must be aperiodic, which means that for any integer $r>1$ there is no integer $p(0<p<r)$ such that $b_{i+p}=b_{i}$ for $i=1, \ldots r-p$. In [5] Coven proves that the topological entropy of this type of $\mathrm{CA}$ is $\log (2)$. Here we consider the Coven $\mathrm{CA}$ with radius $r=2$ and aperiodic word $B=10$. This particular example has the typical behavior of all the other Coven's automata. Let $\mu$ be the uniform measure on $\{0,1\}^{\mathbb{Z}}$. From [3] we know that 000 is a blocking word for $F$. If $\mu$ is the uniform measure $I_{\mu}^{+}+I_{\mu}^{-}=0$ by Proposition 5.2 and $h_{\mu}(F)=0$. On the contrary the sum of the maximum Lyapunov exponents is strictly positive. First it is clear that $\lambda_{\mu}^{+}=0$, because the block map $f$ does not depends on negative coordinates of $x$.

Let $y$ be the fixed point with $y_{i}=1$ for all $i$ and let $z$ be a point with all the coordinates equals to 1 except $z_{0}$. The word 01 never appears in $y$ so $F(y)=$ $y$. Considering that $F(z)(-4,-2)=110$ we deduce that $F^{2}(-6,-4)=110$ 
and by a trivial induction $F^{n}(z)(-2 n-2,-2 n)=110$. Considering that $y$ belong to $S(\mu)=A^{\mathbb{Z}}$ and applying Proposition 3.1 we see that $\lambda_{\mu}^{-} \geq \lambda^{-}(y) \geq$ 2. The value of $\lambda_{\mu}^{-}$must be less than or equal to the radius of the (CA) which is equal to 2 so $\lambda_{\mu}^{-}=2$. It is well known that the topological entropy of the two-shift is equal to $\log 2$. From Theorem 5.1 we get $h_{\mu}(\sigma)\left(\lambda_{\mu}^{+}+\lambda_{\mu}^{-}\right)=$ $2 \log 2>h_{\mu}(F)=0$. Remark that in this case the values of $\lambda_{\mu}^{+}$and $\lambda_{\mu}^{-}$do not allow to prove that $h_{\mu}(F)=0$. From [3] $h_{\text {top }}(F)=2 \log (2)=h_{\mu}(\sigma)\left(\lambda_{\mu}^{+}+\lambda_{\mu}^{-}\right)$ so in this case the inequality of Corollary 5.1 becomes an equality.

\subsection{A sensitive cellular automaton}

Definition 6.1 Let $X$ a compact space and $T$ be a transformation of $X$. The map $T$ is said to be sensitive if there exists a real $\epsilon>0$ such that for any $x \in X$, any real $\delta>0$, there exists a positive integer $n$ and a point $y$ such that $d(x, y)<\delta$ and $d\left(T^{n}(x), T^{n}(y)\right) \geq \epsilon$.

Kuka [12] shows that a cellular automaton is sensitive if and only if it has no equicontinuous points. By Proposition 4.1 sensitive cellular automata have no blocking words, so for all $x \in X$ one has $\lim _{n \rightarrow \infty}\left(I_{n}^{+}(x)+I_{n}^{-}(x)\right)=\infty$.

The aim of studying the sensitive cellular automaton $F$ defined below is twofold. First, in spite of its rather simple behavior, it gives a good idea of the reason why average Lyapunov exponents give a better upper bound of the metric entropy $h_{\mu}(F)$. Secondly, this example shows that inequality (2) is sometimes strict.

Set $X_{1}=\{0,1\}^{\mathbb{Z}}, X_{2}=\{0,1,2\}^{\mathbb{Z}}$ and $X=X_{1} \times X_{2}$. Denote by $\mu_{1}$ the uniform measure on $X_{1}$, by $\mu_{2}$ the uniform measure on $X_{2}$ and $\mu$ the product measure $\mu_{1} \times \mu_{2}$ on $X$. Clearly $\mu$ is the uniform measure on $X$, so $\mu$ is shiftergodic. The cellular automaton $F$ is the product of $F_{1}$ acting on $X_{1}$ and $F_{2}$ acting on $X_{2}$. Denote by $\sigma$ the shift on $X$. The automaton $F_{1}$ is only the shift on $X_{1}$. For each $x \in X_{1}$ one has $\tilde{\Lambda}_{n}^{F_{1}-}(x)=n$. As $\mu_{1}$ is shift-ergodic on $X_{1}$ and $F_{1}$-invariant we can assert that $I_{\mu_{1}}^{-}=\lambda_{\mu_{1}}^{-}=1$.

We define a cellular automaton $F_{2}$ on $X_{2}$ with radius $r$ by its local map $f_{2}$ :

$$
\begin{gathered}
f_{2}\left(x_{-r}, \ldots x_{0}, \ldots x_{r}\right)=x_{0}+x_{r} \text { if } 2 \notin\left\{x_{0}, x_{1}, \ldots, x_{r}\right\} \\
\text { and } f_{2}\left(x_{r}, \ldots x_{0}, \ldots x_{r}\right)=x_{0} \text { if } 2 \in\left\{x_{0}, x_{1}, \ldots, x_{r}\right\} .
\end{gathered}
$$

Using a criterion given in [9, one can easily show that $F_{2}$ is onto, which implies that the product automaton $F$ is also onto. As the uniform measure is 
invariant for an onto cellular automaton the exponents $\left(I_{\mu}^{+}, I_{\mu}^{-}\right)$and $\left(\lambda_{\mu}^{+}, \lambda_{\mu}^{-}\right)$ are defined for $F$ and $\mu$. Remark that for each couple of integers $k$ and $i$ the value of $F^{k}(x)_{i}$ does not depend on the coordinates at the left of $x_{i}$, so for $F$ one has $I_{\mu}^{+}=\lambda_{\mu}^{+}=0$. The letter 2 is clearly a blocking word for $F_{2}$. Every point $x \in X_{2}$ with infinitely many occurrences of 2 in the negative and positive coordinates is an equicontinuous point for $\left(F_{2}, X_{2}\right)$. The restriction of $F_{2}$ to the subshift $\{0,1\}^{\mathbb{Z}}$ is the $r$ times iterated shift. It follows that for $\mu_{2}$-almost all $x$ one has $\Lambda_{n}^{-F_{2}}(x)=r n$, and by Proposition $3.1 \lambda_{\mu_{2}}^{-}=r$. The measure $\mu_{2}$ which is shift-ergodic on $X_{2}$ is also $F_{2}$-invariant because $F_{2}$ is an onto map from $X_{2}$ to $X_{2}$. As $F_{2}$ has equicontinuous points from Proposition 5.2 we have $I_{\mu_{2}}^{-}=0$. From Proposition 5.2 and considering that $S\left(\mu_{2}\right)=X_{2}$ we can compute the value of $\lambda_{\mu_{2}}^{-}$if we find a point $x$ such that $\limsup _{n \rightarrow \infty} \frac{1}{n} \Lambda_{n}^{-}(x)$ be maximum. Denote by $I_{n}^{-F}$ the map $I_{n}^{-}$associated with the automaton $F$ and $I_{n}^{-F_{1}}, I_{n}^{-F_{2}}$ those associated respectively with $F_{1}$ and $F_{2}$. Similarly $\Lambda_{n}^{-F}, \Lambda_{n}^{-F_{1}}$ and $\Lambda_{n}^{-F_{2}}$ are the maps $\Lambda_{n}^{-}$associated with $F$, $F_{1}$ and $F_{2}$. As $F$ is the product of $F_{1}$ by $F_{2}$ we have

$$
I_{n}^{-F}(x)=\max \left\{I_{n}^{-F_{1}}\left(x_{1}\right), I_{n}^{-F_{2}}\left(x_{2}\right)\right\} \text { and } \Lambda_{n}^{-F}(x)=\max \left\{\Lambda_{n}^{-F_{1}}\left(x_{1}\right), \Lambda_{n}^{-F_{2}}\left(x_{2}\right)\right\} .
$$

Remembering that $I_{n, \mu}^{-F}=\int_{X} I_{n}^{-F}(x) d \mu_{1}\left(x_{1}\right) d \mu_{2}\left(x_{2}\right)$ then

$$
I_{n, \mu}^{-F}=\int_{X} \max \left\{I_{n}^{-F_{1}}\left(x_{1}\right), I_{n}^{-F_{2}}\left(x_{2}\right)\right\} d \mu_{1}\left(x_{1}\right) d \mu_{2}\left(x_{2}\right) .
$$

If we consider $F_{2}$ as a map on $X$ we can say that for $\mu_{1} \mu_{2}$-almost all $x \in X$, $I_{n}^{-F_{2}}\left(x_{1}\right)$ is bounded. It follows that $\lim \inf \frac{1}{n} \int_{X} I_{n}^{-F_{2}}(x) d \mu_{1} d \mu_{2}(x)=0$, hence

$$
I_{\mu}^{-}=\liminf \frac{1}{n} \int_{X} I_{n}^{-F_{1}}(x) d \mu_{1} d \mu_{2}(x)=I_{\mu_{1}}^{-}=1 .
$$

If we consider successively $F_{1}$ and $F_{2}$ as maps on $X$, we can see that for $\mu_{1} \mu_{2}$-almost all $x \in X$ we have $\Lambda_{n}^{-F_{1}}(x)=n$ and $\Lambda_{n}^{-F_{2}}(x)=r n$ which implies that

$$
\lambda_{\mu}^{-}=\liminf \int_{X} \max \left\{\Lambda_{n}^{-F_{1}}, \Lambda_{n}^{-F_{2}}\right\} d \mu_{1} d \mu_{2}=\liminf \int_{X} \Lambda_{n}^{-F_{1}} d \mu_{1} d \mu_{2}=I_{\mu_{2}}^{-}=r .
$$

Denoting by $\sigma_{2}$ the shift on $X_{2}$, considering that $h_{\mu}\left(F_{1}\right)=\log 2$ and $h_{\mu}\left(\sigma_{2}\right)=$ $\log 3$ then $h_{\mu}(\sigma)=\log 2+\log 3$. From Proposition 5.2 and taking in account that $F_{2}$ has equicontinuous points we get $h_{\mu_{2}}\left(F_{2}\right)=0$. Considering successively $F_{1}$ and $F_{2}$ as automata on their respective configuration spaces and on $X$ we can assert that

$$
h_{\mu}(F)=h_{\mu}\left(F_{1}\right)+h_{\mu}\left(F_{2}\right)=h_{\mu_{1}}\left(F_{1}\right)+h_{\mu_{2}}\left(F_{2}\right)=\log 2 .
$$


Finally applying inequalities (1) and (2) to $F$, we can conclude that for this example the average Lyapunov exponents give better bounds for the entropy. From Corollary 5.1 one has $h_{\mu}(F)=\log 2 \leq h_{\mu}(\sigma)\left(\lambda_{\mu}^{+}+\lambda_{\mu}^{-}\right)=(\log 2+\log 3) r$ and from Theorem 5.1 we get $h_{\mu}(F)=\log 2 \leq h_{\mu}(\sigma)\left(I_{\mu}^{+}+I_{\mu}^{-}\right)=(\log 2+$ $\log 3)$.

In this example $\mu$ is the uniform measure on $X$ so from Corollary 5.1 the real $h_{\mu}(\sigma)\left(\lambda_{\mu}^{+}+\lambda_{\mu}^{-}\right)=(\log 2+\log 3) r$ is an upper bound of the topological entropy of $F$. The topological entropy of $F$ is the sum of the entropy of $F_{1}$ and $F_{2}$ and is equal to $(r+1) \log (2)$ which means that in this case the topological inequality is strict.

Remark 6 It will be interesting to find no trivial examples for which inequality (2) becomes an equality and with a strict inequality (1). The Proposition 5.2 suggest that we know very little about sensitive cellular automata. The condition $h_{\mu}(\sigma)\left(I_{\mu}^{+}+I_{\mu}^{-}\right)>0$ does not imply that $h_{\mu}(F)>0$.

I am indebted to François Blanchard for many stimulating conversations and for his help for the writing. An important part of this work has been done in the University of Chile in Santiago, Mathematics's laboratory of the section Civil Engineering. I want to thanks "FONDAP en Matematicas Aplicadas, proyecto Modelamiento Estocastico" and ECOS for the financial support and Alejandro Maass for his numerous suggestions.

\section{References}

[1] M. Brin And A. Katok (1983) , On local entropy, Lecture Notes in Math., 470, Springer-Verlag, New York, 30-38.

[2] F. Blanchard, A. MaAss, Ergodic properties of expansive one-sided cellular automata, Israel J. Math. 99, 149-174 (1997).

[3] F. Blanchard, A. MaAss, Dynamical behavior of Coven' aperiodic cellular automata, Theor . Computer Sci. 163, 291-302 (1996).

[4] F. Blanchard, P. Tisseur, Some properties of cellular automata with equicontinuity points, Ann. Inst. Henri Poincaré, Probabilité et Statistiques 36,5 569-582 (2000). 
[5] E. M. Coven, Topological entropy of block maps, Proc. Amer. Math. Soc. 78, Number 4, (1980).

[6] E. Coven And M. Paul, Endomorphisms of Irreducible Subshifts of Finite Type, Math. Systems Th., Vol 8, No.2 Springer-Verlag (1974).

[7] M. Denker, C. Grillenberger, K. Sigmund, Ergodic theory on compact spaces, Lecture Notes 527,Springer, Berlin (1975).

[8] R. H. Gilman, Classes of linear automata, Ergodic Th. Dynam. Syst., 7, 105-118 (1987).

[9] G. A. Hedlund, Endomorphisms and Automorphisms of the Shifts Dynamical System, Math. Systems Th. 3, 320-375 (1969).

[10] M. Hurley, Attractors in cellular automata, Ergodic Th. and Dynam. Syst., 10, 131-140, (1990).

[11] J.P.C Kingman (1973), Subadditive ergodic theory, Ann.Proba. 1, 883-909.

[12] P. KuRKA, Languages, equicontinuity and attractors in linear cellular automata, Ergod. Th. Dynam. Syst. 217, 417-433 (1997).

[13] D. A. Lind, Applications of ergodic theory and sofic systems to cellular automata, Physica 10D, 36-44, (1984).

[14] K. Petersen, Ergodic theory, Cambrige studies in advance mathematics 2 .

[15] M. Pollicott, Lectures on ergodic theory and Pesin theory on compact manifolds, Cambridge University Press.

[16] D. Ruelle, Ergodic theory of differentiable dynamical systems, Publ. Math.IHES 275-306 (1979).

[17] M.A. Shereshevsky, Lyapunov Exponents for One-Dimensional Cellular Automata. J. Nonlinear Sci. Vol.2, 1-8 (1991).

[18] M.A. Shereshevsky, Ergodic properties of Certain Surjective Cellular Automata. Monats. Math. 114, 305-316 (1992). 
[19] P. Tisseur, Average Lyapunov exponents and one dimensional cellular automata, Prétirage de l'IML n 2000-12.

[20] P. Walters, An introduction to ergodic theory, Graduate texts in Mathematics, Springer, Berlin, (1979).

[21] S. Wolfram, Theory and Applications of Cellular Automata, World Scientific, (1986). 